\title{
Numerical Calculation for Error Formula for Boole's Rule
}

Roaa A. Fadhil

University, Iraq Ministry of Education, General Directorate of Education Qadsiyah, Iraq

Corresponding Author Email: roaaazizfadhil@gmail.com

https://doi.org/10.18280/ama_a.562-405

Received: 18 September 2019

Accepted: 5 December 2019

\section{Keywords:}

bool's rule, Romberg integral, singular

integrands

\begin{abstract}
The main aim of this paper is to calculate the value of integration which Singular Integrands or have Integrands with Singular derivatives at one end (or both ends) of the integration region by deriving errors formula (correction terms) using Composite Boole's rule and Romberg integral to improve the results.
\end{abstract}

\section{INTRODUCTION}

Many researchers have worked to find the values Numerical integration $[1,2]$, and of them work in particular to find of continuous and Improper integrations let us mention just a few Fox [3], Fox and Linda [4], Wu and Liu [5] and Davis and Rabinowitz [6] studied the integrals its integrands singularity but neglects the singularity introduced to derive errors formula (correction terms) by using composite Boole rule $[7,8]$ to calculate an approximate values of integrals have its integrands have singular derivatives and singular integrals at end of region of integration and we will use Romberg integration (acceleration) $[9,10]$ to improve the results.

Let $\boldsymbol{I}$ is integral

$$
I=\int_{a}^{b} f(x) d x
$$

It can calculate in approximation by Composite Boole's rules

$$
I=\int_{a}^{b} f(x) d x \cong B(h)+E_{B}
$$

where, $B(h)$ is composite Boole's rule is defined.

Consider a $f(x)$ over interval $[a, b]$

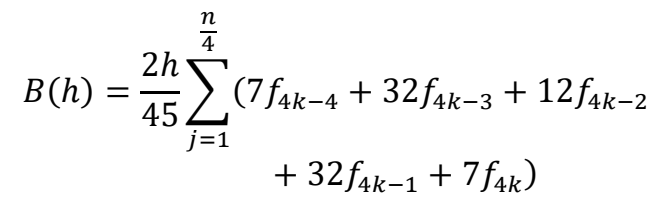

where, $n=4 k, k \geq 1$, that is $n$ is restricted to be a multiple of 4 , $x_{j}=a+j h h=\frac{(b-a)}{n}$, and $f_{j}=f\left(x_{j}\right), j=0,1,2, \ldots, n$.

$E_{B}$ error formula for the Boole rule given by

$$
E_{B}=-\frac{2 h^{6}(b-a)}{945} f^{6}(\eta)+-\frac{2 h^{8}(b-a)}{900} f^{8}(\eta)
$$

where, for some $\eta$ in $[a, b][7]$

We can use Romberg Integration will write the rule with the error formulas as follows

$$
I-B(h)=A_{1} h^{6}+A_{2} h^{8}+\cdots
$$

where, $A_{i}, i=1,2,3, \ldots$ is coefficients [8].

\section{MAIN RESULTS}

\subsection{Integrals for Integrands with Singularity Derivatives Points}

Theorem (1):

Let the function $f(x)$ is continuous and differentiable at each point of the region of integrals $[a, b]$, but at least one of the derivatives at the point $(a)$, The approximate value of integration can be calculated from the following rule

$$
I=\int_{a}^{b} f(x) d x \cong B(h)+E_{B}(h)
$$

\section{Such that}

$$
E_{B}(h)=\left[a_{1} h^{7} D^{6}+a_{1} h^{8} D^{7}+a_{1} h^{9} D^{8}+\cdots\right] f\left(x_{3}\right)+
$$
$A_{1} h^{6}+A_{2} h^{8}+\cdots$

$A_{i}, a_{i}, i=1,2,3, \ldots$ are constants depend on the derivatives of the function $f(x)$ on the integral region and $B(h)$ Similar to the formula (3), and $x_{j}=a+j h$,

$$
j=0,1,2, \ldots, n
$$

Proof: we can write the integral $I$ in the form

$$
\begin{aligned}
I=\int_{a}^{b} f(x) d x & =\int_{x_{0}}^{x_{n}} f(x) d x=\int_{x_{0}}^{x_{4}} f(x) d x \\
& +\int_{x_{4}}^{x_{n}} f(x) d x
\end{aligned}
$$


For the first integration is defined at each point of the integrations but its derivatives are not defined at the point $x_{0}$ in the partial region $\left[x_{0}, x_{4}\right]$ we using (Taylor's series [11]) for the function $f(x)$ about the point $x_{4}$ we get

$$
\begin{aligned}
f(x)=f\left(x_{4}\right)+ & \left(x-x_{4}\right) f^{\prime}\left(x_{4}\right) \\
& +\frac{\left(x-x_{4}\right)^{2}}{2 !} f^{\prime \prime}\left(x_{4}\right) \\
& +\frac{\left(x-x_{4}\right)^{3}}{3 !} f^{\prime \prime \prime}\left(x_{4}\right) \\
& +\frac{\left(x-x_{4}\right)^{4}}{4 !} f^{4}\left(x_{4}\right) \\
& +\frac{\left(x-x_{4}\right)^{5}}{5 !} f^{5}\left(x_{4}\right) \\
& +\frac{\left(x-x_{4}\right)^{6}}{6 !} f^{6}\left(x_{4}\right)+\cdots
\end{aligned}
$$

We suppose that all partial derivatives to $f(x)$ exist at the point $\left(x_{4}\right)$, By taking the integral for formula (7) in the region $\left[x_{0}, x_{4}\right]$ we obtain

$$
\begin{aligned}
I_{1}=\int_{x_{0}}^{x_{4}} f(x)= & 4 h f\left(x_{4}\right)-8 h^{2} f^{\prime}\left(x_{4}\right) \\
& +\frac{32 h^{3}}{3} f^{\prime \prime}\left(x_{4}\right)-\frac{32 h^{4}}{3} f^{\prime \prime \prime}\left(x_{4}\right) \\
& +\frac{128 h^{5}}{15} f^{4}\left(x_{4}\right)-\frac{4^{4} h^{6}}{45} f^{5}\left(x_{4}\right) \\
& +\frac{4^{5} h^{7}}{315} f^{6}\left(x_{4}\right)+\cdots
\end{aligned}
$$

From formula (7) we get

(1) $f\left(x_{0}\right)=f\left(x_{4}\right)-4 h f^{\prime}\left(x_{4}\right)+8 h^{2} f^{\prime \prime}\left(x_{4}\right)-$ $\frac{32 h^{3}}{3} f^{\prime \prime \prime}\left(x_{4}\right)+\frac{32 h^{4}}{3} f^{4}\left(x_{4}\right)-\frac{128 h^{5}}{15} f^{5}\left(x_{4}\right)+\frac{4^{4} h^{6}}{45} f^{6}\left(x_{4}\right)+$

(2) $f\left(x_{0}+h\right)=f\left(x_{4}\right)-3 h f^{\prime}\left(x_{4}\right)+\frac{9}{2} h^{2} f^{\prime \prime}\left(x_{4}\right)-$ $\frac{9 h^{3}}{2} f^{\prime \prime \prime}\left(x_{4}\right)+\frac{27 h^{4}}{8} f^{4}\left(x_{4}\right)-\frac{81 h^{5}}{40} f^{5}\left(x_{4}\right)+\frac{81 h^{6}}{80} f^{6}\left(x_{4}\right)+\cdots$

(3) $f\left(x_{0}+2 h\right)=f\left(x_{4}\right)-2 h f^{\prime}\left(x_{4}\right)+2 h^{2} f^{\prime \prime}\left(x_{4}\right)-$ $\frac{4 h^{3}}{3} f^{\prime \prime \prime}\left(x_{4}\right)+\frac{2 h^{4}}{3} f^{4}\left(x_{4}\right)-\frac{4 h^{5}}{15} f^{5}\left(x_{4}\right)+\frac{4 h^{6}}{45} f^{6}\left(x_{4}\right)+\cdots$

(4) $f\left(x_{0}+3 h\right)=f\left(x_{4}\right)-h f^{\prime}\left(x_{4}\right)+\frac{1}{2} h^{2} f^{\prime \prime}\left(x_{4}\right)-$ $\frac{h^{3}}{6} f^{\prime \prime \prime}\left(x_{4}\right)+\frac{h^{4}}{24} f^{4}\left(x_{4}\right)-\frac{h^{5}}{120} f^{5}\left(x_{4}\right)+\frac{h^{6}}{720} f^{6}\left(x_{4}\right)+\cdots$

from (8) and (1), (2), (3), (4) and

$f\left(x_{4}\right)=E f\left(x_{3}\right)=f\left(x_{0}+3 h\right)$ we get $E=e^{h D}$ where, $D f(x)=f^{\prime}(x)$. [10]

$$
\begin{gathered}
I_{1}=\int_{x_{0}}^{x_{4}} f(x) d x=\frac{2 h}{45}\left[7 f\left(x_{0}\right)+32 f\left(x_{1}\right)\right. \\
+12 f\left(x_{2}\right) \\
\left.+32 f\left(x_{3}\right)+7 f\left(x_{4}\right)\right]+\left[\frac{1532}{21} h^{7} f^{6}\left(x_{4}\right)+\cdots\right] \\
E f\left(x_{3}\right) \ldots \\
I_{1}=\int_{x_{0}}^{x_{4}} f(x) d x=\frac{2 h}{45}\left[7 f\left(x_{0}\right)+32 f\left(x_{1}\right)\right. \\
\left.+12 f\left(x_{2}\right)\right] \\
\left.+32 f\left(x_{3}\right)+7 f\left(x_{4}\right)\right] f\left(x_{3}\right)+\left[a_{1} h^{7} D^{6}+a_{2} h^{8} D^{7}\right. \\
\left.++a_{3} h^{9} D^{8}+\ldots\right] \ldots
\end{gathered}
$$

Such that $a_{i}$ is constants, for all , $i=1,2,3, \ldots$

And the second integral is continuous and differentiable at each point of the region of integrals $\left[x_{4}, x_{n}\right]$

$$
\begin{gathered}
I_{2}=\int_{x_{4}}^{x_{n}} f(x) d x=\frac{2 h}{45} \sum_{j=2}^{\frac{n}{4}}\left(7 f\left(x_{4 j-4}\right)\right. \\
+32 f\left(x_{4 j-3}\right) \\
\left.+12 f\left(x_{4 j-2}\right)+32 f\left(x_{4 j-1}\right)+7 f_{\left(x_{4 j}\right)}\right)+A_{1} h^{6} \\
+A_{2} h^{8}+\cdots
\end{gathered}
$$

Such that $A_{i}$ is constants, for all , $i=1,2,3, \ldots$ from formulas (10), (11) we get

$$
\begin{gathered}
I=\int_{x_{0}}^{x_{n}} f(x) d x=\frac{2 h}{45} \sum_{j=1}^{\frac{n}{4}}\left(7 f\left(x_{4 j-4}\right)+32 f\left(x_{4 j-3}\right)\right. \\
\left.+12 f\left(x_{4 j-2}\right)+32 f\left(x_{4 j-1}\right)+7 f_{\left(x_{4 j}\right)}\right)+ \\
A_{1} h^{6}+A_{2} h^{8}+\cdots
\end{gathered}
$$

\section{Theorem (2)}

Let the function $f(x)$ is continuous and differentiable at each point of the region of integrals $[a, b]$, at least one of the derivatives at the point (b), The approximate value of integration can be calculated from the following rule.

$$
\begin{gathered}
I=\int_{a}^{b} f(x) d x=B(h)+E_{B}(h) \\
E_{B}(h)=\left[c_{1} h^{7} D^{6}+c_{2} h^{8} D^{7}+c_{3} h^{9} D^{8}+\ldots\right] f\left(x_{n-1}\right)
\end{gathered}
$$

Such that $A_{i}, c_{i}, i=1,2,3, \ldots$ are constants depend on the derivatives of the function $f(x)$ on the integral region and $B(h)$ Similar to the formula (3), and $x_{j}=a+j h, j=0,1,2, \ldots$

Proof: we can write the integral $I$ in the form

$$
\begin{aligned}
I=\int_{a}^{b} f(x) d x & =\int_{x_{0}}^{x_{n}} f(x) d x=\int_{x_{0}}^{x_{n-4}} f(x) d x \\
& +\int_{x_{n-4}}^{x_{n}} f(x) d x \ldots
\end{aligned}
$$

For the first integration is defined at each point of the integrations we get

$$
\begin{aligned}
& I_{1}=\int_{x_{0}}^{x_{n-4}} f(x) d x=\frac{2 h}{45} \sum_{j=1}^{\left.\frac{(n}{4}\right)-4}\left(7 f\left(x_{4 j-4}\right)\right. \\
& +32 f\left(x_{4 j-3}\right)+12 f\left(x_{4 j-2}\right)+32 f\left(x_{4 j-1}\right) \\
& +7 f\left(x_{4 j}\right)+A_{1} h^{6}+A_{2} h^{8}+\cdots
\end{aligned}
$$

Such that $A_{i}$ is constants, for all, $i=1,2,3, \ldots$

And the second integral but its partial derivatives are not defined at the point $x_{4}$ in the partial region $\left[x_{4}, x_{n-4}\right]$ we using Taylor's series for the function $f(x)$ about the point $\left(x_{n-4}\right)$

$$
\begin{gathered}
f(x)=f\left(x_{n-4}\right)+\left(x-x_{n-4}\right) f^{\prime}\left(x_{n-4}\right)+ \\
\frac{\left(x-x_{n-4}\right)^{2}}{2 !} f^{\prime \prime}\left(x_{n-4}\right)+\frac{\left(x-x_{n-4}\right)^{3}}{3 !} f^{\prime \prime \prime}\left(x_{n-4}\right)
\end{gathered}
$$




$$
\begin{aligned}
& +\frac{\left(x-x_{n-4}\right)^{4}}{4 !} f^{4}\left(x_{n-4}\right) \\
& +\frac{\left(x-x_{n-4}\right)^{5}}{5 !} f^{5}\left(x_{n-4}\right) \\
& +\frac{\left(x-x_{n-4}\right)^{6}}{6 !} f^{6}\left(x_{n-4}\right)+\cdots
\end{aligned}
$$

We suppose that all derivatives to $f(x)$ exist at the point $\left(x_{n-4}\right)$, By taking the integral for formula (7) in the region we obtain

$$
\begin{aligned}
I_{2}=\int_{x_{0}}^{x_{4}} f(x) d x & =4 h f\left(x_{4}\right)+8 h^{2} f^{\prime}\left(x_{4}\right) \\
& +\frac{32 h^{3}}{3} f^{\prime \prime}\left(x_{4}\right) \\
+\frac{-32 h^{4}}{3} f^{\prime \prime \prime}\left(x_{4}\right) & +\frac{128 h^{5}}{15} f^{4}\left(x_{4}\right) \\
+ & \frac{4^{4} h^{6}}{45} f^{5}\left(x_{4}\right)+\frac{4^{5} h^{7}}{315} f^{6}\left(x_{4}\right) \\
+\cdots & \ldots
\end{aligned}
$$

From formula (14) we get

(1) $f\left(x_{n}\right)=f\left(x_{n-4}\right)+4 h f^{\prime}\left(x_{n-4}\right)+8 h^{2} f^{\prime \prime}\left(x_{n-4}\right)+$ $\frac{32 h^{3}}{3} f^{\prime \prime \prime}\left(x_{n-4}\right)+\frac{32 h^{4}}{3} f^{4}\left(x_{n-4}\right)+\frac{128 h^{5}}{15} f^{5}\left(x_{n-4}\right)+$ $\frac{4^{4} h^{6}}{45} f^{6}\left(x_{n-4}\right)+\cdots$

(2) $f\left(x_{n}-h\right)=f\left(x_{n-4}\right)+3 h f^{\prime}\left(x_{n-4}\right)+$ $\frac{9}{2} h^{2} f^{\prime \prime}\left(x_{n-4}\right)+\frac{9 h^{3}}{2} f^{\prime \prime \prime}\left(x_{n-4}\right)+\frac{27 h^{4}}{8} f^{4}\left(x_{n-4}\right)+$ $\frac{81 h^{5}}{40} f^{5}\left(x_{n-4}\right)+\frac{81 h^{6}}{80} f^{6}\left(x_{n-4}\right)+\cdots$

(3) $f\left(x_{n}-2 h\right)=f\left(x_{n-4}\right)+2 h f^{\prime}\left(x_{n-4}\right)+$ $2 h^{2} f^{\prime \prime}\left(x_{n-4}\right)+\frac{4 h^{3}}{3} f^{\prime \prime \prime}\left(x_{n-4}\right)+\frac{2 h^{4}}{3} f^{4}\left(x_{n-4}\right)+$ $\frac{4 h^{5}}{15} f^{5}\left(x_{n-4}\right)+\frac{4 h^{6}}{45} f^{6}\left(x_{n-4}\right)+\cdots$

(4) $f\left(x_{0}-3 h\right)=f\left(x_{n-4}\right)+h f^{\prime}\left(x_{x_{n-4}}\right)+$ $\frac{1}{2} h^{2} f^{\prime \prime}\left(x_{n-4}\right)+\frac{h^{3}}{6} f^{\prime \prime \prime}\left(x_{n-4}\right)+\frac{h^{4}}{24} f^{4}\left(x_{n-4}\right)+$ $\frac{h^{5}}{120} f^{5}\left(x_{n-4}\right)+\frac{h^{6}}{720} f^{6}\left(x_{n-4}\right)+\cdots$

from (15) and (1), (2), (3), (4) and

$E^{-1} f\left(x_{n}\right)=f\left(x_{n-1}\right)=f\left(x_{n}-h\right)$ we get $E^{-1}=e^{-h D}$ where $D f(x)=f^{\prime}(x)$

$$
\begin{aligned}
& I_{2}=\int_{x_{0}}^{x_{4}} f(x) d x=\frac{2 h}{45}\left[7 f\left(x_{0}\right)+32 f\left(x_{1}\right)\right. \\
&\left.+12 f\left(x_{2}\right)+32 f\left(x_{3}\right)+7 f\left(x_{4}\right)\right] \\
&+\left[\frac{1532}{21} h^{7} D^{6}+\cdots\right] E^{-1} f\left(x_{n-1}\right) \\
& I_{2}=\int_{x_{0}}^{x_{4}} f(x)= \frac{2 h}{45}\left[7 f\left(x_{0}\right)+32 f\left(x_{1}\right)+12 f\left(x_{2}\right)\right. \\
&\left.+32 f\left(x_{3}\right)+7 f\left(x_{4}\right)\right] \\
&+\left[c_{1} h^{7} D^{6}+c_{2} h^{8} D^{7}+c_{3} h^{9} D^{8}\right. \\
&+\cdots] f\left(x_{n-1}\right) \cdots
\end{aligned}
$$

$c_{i}, i=1,2,3, \ldots$ is constants

from formulas (13), (17) we get

$$
\begin{gathered}
I=\int_{x_{0}}^{x_{n}} f(x) d x=\frac{2 h}{45} \sum_{j=1}^{\frac{n}{4}}\left(7 f\left(x_{4 j-4}\right)+32 f\left(x_{4 j-3}\right)\right. \\
\left.+12 f\left(x_{4 j-2}\right)+32 f\left(x_{4 j-1}\right)+7 f_{\left(x_{4 j}\right)}\right)+\left[c_{1} h^{7} D^{6}+\right. \\
\left.c_{2} h^{8} D^{7}+c_{3} h^{9} D^{8}+\cdots\right] f\left(x_{n-1}\right)+A_{1} h^{6}+A_{2} h^{8}+\cdots
\end{gathered}
$$

Corollary: Let the function $f(x)$ is continuous and differentiable at each point of the region of integrals $[a, b]$, but at least one of the derivatives at the point the points $(a, b)$, The approximate value of integration can be calculated from the following rul

$$
\begin{gathered}
I=\int_{a}^{b} f(x) d x \cong B(h)+E_{B}(h) \\
E_{B}(h)=\left[b_{1} h^{7} D^{6}+b_{2} h^{8} D^{7}+b_{3} h^{9} D^{8}+\cdots\right] f\left(x_{3}\right) \\
+\left[c_{1} h^{7} D^{6}+c_{2} h^{8} D^{7}+c_{3} h^{9} D^{8}\right. \\
+\cdots] f\left(x_{n-1}\right)+A_{1} h^{6}+A_{2} h^{8}+\cdots
\end{gathered}
$$

Such that $A_{i}, a_{i}, c_{i}$, for all, $i=1,2,3, \ldots$ are constants depend on the partial derivatives of the function $f(x)$ on the integral region and $B(h)$ Similar to the formula (3), and $x_{j}=$ $a+j h, j=0,1,2, \ldots$.

Proof: Can be easily using a theorem (1), (2)

\subsection{Integrals with Singular integrands}

The function is continuous and derivable in the integration region $[a, b]$ but not defined at the point a. Here we cannot calculate the value of integration using the theorem (2-1), because it uses the value of the integral in the point and to obtain the value of integration using the above theorem, Davies and Rabinowitz [6] and calculating the error formulas of them, as well as if they are not defined in the point or both points are not defined

\section{EXAMPLES}

(1) $\int_{0}^{1} x^{\frac{3}{2}} d x$ which analytical value is 0.4 approximate to 14 decimal places the integrand which has singula in the second derivative $\left(x_{0}=0\right)$ and the formula of the correction terms $\left(A_{1} h^{2.5}+A_{2} h^{8}+A_{3} h^{10}+A_{4} h^{12}+\cdots\right)$ Such that $A_{i}$, $i=1,2,3, \ldots$ are constants from the table (1) The value of the integration is correct for six decimal places using a base Boole, while using the Romberg integral method with the above rule, we note that the value equal to the analytical value when ( subintervals $n=64$ ) 
Table 1

\begin{tabular}{clcccc}
\hline $\mathbf{n}$ & Bool values & $\boldsymbol{h}^{\mathbf{2 . 5}}$ & $\boldsymbol{h}^{\mathbf{6}}$ & $\boldsymbol{h}^{\mathbf{8}}$ & $\boldsymbol{h}^{\mathbf{1 0}}$ \\
\hline 4 & 0.40030278197718 & & & & \\
8 & 0.40005360500749 & 0.40000009743428 & & & \\
16 & 0.40000947773754 & 0.40000000196966 & 0.40000000045435 & & \\
32 & 0.40000167547077 & 0.40000000003359 & 0.40000000000286 & 0.40000000000109 & \\
64 & 0.40000029618463 & 0.40000000000054 & 0.40000000000001 & 0.40000000000000 & 0.40000000000000 \\
\hline
\end{tabular}

(2) $\int_{0}^{1}(1-x)^{\frac{-1}{4}} d x$ which analytical value is 1.33333333333333 approximate to 14 decimal places To calculate the integration numerically note that the integrand at has singular point at the upper end $\left(x_{n}=1\right)$ the error formula is $\left(t_{1} h^{0.75}+t_{2} h^{8}+t_{3} h^{10}+t_{4} h^{12}+\cdots\right)$ Such that $t_{i}, i=$
$1,2,3, \ldots$ are constants from the table (2) The value of the integration is correct for one decimal places using a base Boole, while using the Romberg integral method with the above rule, we note that the value equal to the analytical value when ( subintervals $n=128$ )

Table 2

\begin{tabular}{clccccc}
\hline $\mathbf{n}$ & Bool values & $\boldsymbol{h}^{\mathbf{0 . 7 5}}$ & $\boldsymbol{h}^{\mathbf{6}}$ & $\boldsymbol{h}^{\mathbf{8}}$ & $\boldsymbol{h}^{\mathbf{1 0}}$ & $\boldsymbol{h}^{\mathbf{1 2}}$ \\
\hline 4 & 1.12123952438139 & & & & & \\
8 & 1.20722309696577 & 1.33333702591451 & & & & \\
16 & 1.25834777436493 & 1.33333342266093 & 1.33333336546643 & & & \\
32 & 1.28874665387441 & 1.33333333497350 & 1.33333333358163 & 1.33333333345660 & & \\
64 & 1.30682193512078 & 1.33333333336021 & 1.33333333333461 & 1.33333333333364 & 1.333333333333352 & \\
128 & 1.31756956164199 & 1.33333333333376 & 1.33333333333334 & 1.33333333333333 & 1.33333333333333 & 1.33333333333333 \\
\hline
\end{tabular}

(3) $\int_{0}^{1}-x^{2} \ln (x) d x$ which analytical value is 0.111111111111111 approximate to 15 decimal places to calculate the integration numerically note that the integrand at has singular point at the lower end $\left(x_{0}=0\right)$ and the error formulas for this integration is $\left(d_{1} h^{3}+d_{2} h^{6}+d_{3} h^{8}+\right.$ $\left.d_{4} h^{10}+\cdots\right)$ Such that $d_{i}, i=1,2,3, \ldots$ are constants from the table (3) The value of the integration is correct for seven decimal places using a base Boole, while using the Romberg integral method with the above rule, we note that the value equal to the analytical value when ( subintervals $n=128$ ).

Table 3

\begin{tabular}{ccccccc}
\hline $\boldsymbol{n}$ & Bool values & $\boldsymbol{h}^{\mathbf{3}}$ & $\boldsymbol{h}^{\mathbf{6}}$ & $\boldsymbol{h}^{\mathbf{8}}$ & $\boldsymbol{h}^{\mathbf{1 0}}$ & $\boldsymbol{h}^{\mathbf{1 2}}$ \\
\hline 4 & 0.111447861867241 & & & & & \\
8 & 0.111153371033969 & 0.111111300914930 & & & & \\
16 & 0.111116396808825 & 0.111111114776662 & 0.111111102367444 & & & \\
32 & 0.111111771877083 & 0.111111111172548 & 0.111111110932274 & 0.111111111068223 & & \\
64 & 0.111111193707714 & 0.111111111112090 & 0.111111111108059 & 0.111111111110850 & 0.111111111111017 & \\
128 & 0.111111121435700 & 0.1111111111111126 & 0.1111111111111062 & 0.1111111111111110 & 0.1111111111111111 & 0.1111111111111111 \\
\hline
\end{tabular}

(4) $\int_{0}^{1} \sqrt{x} e^{x} d x \quad$ which analytical value is 0.12556300825518 approximate to 13 decimal places To calculate the integration numerically note that the integrand at has singular point at the lower end $\left(x_{0}=0\right)$ and the error formulas for this integration is $\left(j_{1} h^{1.5}+j_{2} h^{2.5}+j_{3} h^{3.5}+\right.$ $\left.j_{4} h^{4.5}+j_{5} h^{5.5}+j_{6} h^{6}+j_{7} h^{6.5}+\cdots\right)$ Such that $d_{i}, i=$
$1,2,3, \ldots$ are constants from the table (4) The value of the integration is correct for four decimal places using a base Boole, while using the Romberg integral method with the above rule, we note that the value equal to the analytical value when ( subintervals $n=256$ ).

Table 4

\begin{tabular}{|c|c|c|c|c|c|c|c|}
\hline $\mathbf{n}$ & Bool values & $h^{1.5}$ & $h^{2.5}$ & $h^{3.5}$ & $h^{4.5}$ & $h^{5.5}$ & $h^{6}$ \\
\hline 4 & 1.2470029863351 & & & & & & \\
\hline 8 & 1.2525311205590 & 1.2555545575607 & & & & & \\
\hline 16 & 1.2545254859732 & 1.2556162406373 & 1.2556294862905 & & & & \\
\hline 64 & 1.2554911416387 & 1.2556296313923 & 1.2556300744615 & 1.2556300816319 & 1.2556300830775 & & \\
\hline 128 & 1.2555809075678 & 1.2556300021847 & 1.2556300818076 & 1.2556300825199 & 1.2556300825610 & 1.2556300825493 & \\
\hline 256 & 1.2556126873498 & 1.2556300682897 & 1.2556300824849 & 1.2556300825506 & 1.2556300825520 & 1.2556300825518 & 1.2556300825518 \\
\hline
\end{tabular}

\section{THE DISCUSSION}

It is clear according to tables of results of this research that when we calculate the approximate value for the integration when integrands with singular derivatives and Singular Integrands with by the composite Boole rule when we are using Romberg accelerating Using the calculated errors formula with rule we can get a best results with respect to convergence to value of integration with a few number from subintervals Thus, we can depend on method in a calculation the integrals 


\section{REFERENCES}

[1] Jones, L.K. (2017). An Elementary derivation of the numerical integration bounds in beginning calculus. Mathematical Association of America, 124(6): 558-561.

[2] Hota, M. K., Saha, A. K., Ojha, P., \& Mohanty, P. K. (2017). On the approximate evaluation of oscillatorysingular integrals. Cogent Mathematics, 4(1): 1314066. https://doi.org/10.1080/23311835.2017.1314066

[3] Fox, L. (1967). Romberg integration for a class of singular integrands. The Computer Journal, 10(1): 87-93. https://doi.org/10.1093/comjnl/10.1.87

[4] Fox, L., Hayes, L. (1970). On the definite integration of singular integrands. SIAM Review, 12(3): 449-457. https://doi.org/10.1137/1012084

[5] Wu, S.F., Liu, X. (2016). Asymptotic expressions for the error terms of two Newton-Cotes quadrature formulae. Journal of Interdisciplinary Mathematics, 19(5\&6): 1025-1037.

https://doi.org/10.1080/09720502.2016.1220095
[6] Davis, P.J., Rabinowitz, P. (1975). Methods of Numerica Integration. Blasdell Puplishing Company, Chapter 5.

[7] Al-Alaoui, M.A. (1996). A class of numerical integration rules with first-order derivatives. ACM Signum Newslett, 31(2): 25-44. https://doi.org/10.1145/230922.230930

[8] Mathews, J.H., Fink, K.D. (2004). Numerical Methods using Matlab. 4th Ed., Pearson Prentice Hall.

[9] Canale, R.P., Chapra, S.C. (2010). Numerical methods $f$ or engineer. Sixed edition, McGraw-Hill, New york, 601-648.

[10] Rozema, E.R. (2018). Romberg integration by taylor series. TheAmerican Mathematical Monthly, 94(3): 284288. https://doi.org/10.1080/00029890.1987.12000629

[11] Sastry, S.S. (2012). Introductory Methods of Numerical Analysis. New Delhi, Fourth Edition.

[12] Yang, Y.J., Gordon, S.P. (2016). Numerical Integration: One Step at a Time. PRIMUS, 26(5): 371-392. https://doi.org/10.1080/10511970.2015.1127300 